Also available at http://amc.imfm.si

ISSN 1855-3966 (printed edn.), ISSN 1855-3974 (electronic edn.)

ARS MATHEMATICA CONTEMPORANEA 2 (2009) 49-58

\title{
Edge-contributions of some topological indices and arboreality of molecular graphs
}

\author{
Tomaž Pisanski \\ Faculty of Mathematics and Physics, University of Ljubljana, \\ University of Primorska and \\ Institute of Mathematics, Physics, and Mechanics \\ Jadranska 19, 1000 Ljubljana, Slovenia \\ Janez Žerovnik \\ Faculty of Mechanical Engineering, University of Ljubljana and \\ Institute of Mathematics, Physics, and Mechanics \\ Jadranska 19, 1000 Ljubljana, Slovenia
}

Received 27 June 2008, accepted 20 January 2009, published online 20 March 2009

\begin{abstract}
Some graph invariants can be computed by summing certain values, called edge-contributions over all edges of graphs. In this note we use edge-contributions to study relationships among three graph invariants, also known as topological indices in mathematical chemistry: Wiener index, Szeged index and recently introduced revised Szeged index. We also use the quotient between the Wiener index and the revised Szeged index to study arboreality (tree-likeness) of graphs.
\end{abstract}

Keywords: Mathematical chemistry, topological index, revised Szeged index.

Math. Subj. Class.: 05C19, 05C90

\section{Introduction and Motivation}

In mathematical chemistry some graph invariants are being studied intensively since they correlate well, when applied to molecular graphs, with certain properties of the corresponding molecules [16]. In this note we explore three such invariants, all based on the Wiener index [17], that was initially defined for trees and admits several non-equivalent generalizations to general graphs.

E-mail addresses: Tomaz.Pisanski@fmf.uni-lj.si (Tomaž Pisanski), Janez.Zerovnik@imfm.uni-lj.si (Janez Žerovnik) 
Traditionally, the Wiener index for general graphs is defined as the sum of all distances in a graph. Ivan Gutman [6] introduced another generalization that is known under the name of Szeged index. Recently Milan Randić modified the definition of the Szeged index. The new index was named revised Szeged index by Pisanski and Randić [12].

Let $G$ be any connected graph. Then one can define the usual distance function on its vertex set $V(G)$. Namely, $d(u, v)$ is the number of edges on any of the shortest paths joining vertex $u$ to vertex $v$. The Wiener index is defined as:

$$
W(G)=(1 / 2) \sum_{(u, v) \in V(G) \times V(G)} d(u, v)
$$

where the sum runs over all ordered pairs of vertices. The factor $(1 / 2)$ is needed in order to count each pair exactly once. If we want to avoid extra work, it is more convenient to consider unordered pairs. For example, if the vertex set is linearly ordered, we can write

$$
W(G)=\sum_{u<v, u, v \in V(G)} d(u, v) .
$$

Define

$$
W(u, v)=\{x \in V(G) \mid d(u, x)<d(v, x)\} .
$$

Let $w(u, v)$ denote the number of vertices that are closer to $u$ than to $v$, i.e. $w(u, v):=$ $|W(u, v)|$. Therefore, $w(v, u)$ is the number of vertices that are closer to $v$ than to $u$ : $w(v, u):=|W(v, u)|$.

Proposition 1.1. For any connected graph $G$ and any pair of distinct vertices $u$ and $v$ the sets $W(u, v)$ and $W(v, u)$ are non-empty and disjoint.

We may define: $O(u, v):=V(G)-W(u, v)-W(v, u)$. Clearly, $O(u, v)=O(v, u)$ and the sets $O(u, v), W(u, v), W(v, u)$ form the so-called fundamental partition of the vertex set $V(G)$. Note that sometimes these three sets are denoted by ${ }_{u} W_{v}, W_{u v}, W_{v u}$, respectively; see, for instance [8].

Let $T$ be a tree. Let $e=u \sim v$ be any of its edges joining adjacent vertices $u$ and $v$. The Wiener index $W(T)$ of $T$ can be computed as

$$
W(T)=\sum_{u \sim v \in E(G)} w(u, v) w(v, u)
$$

based on the following theorem (that was known already to Wiener [17]).

Theorem 1.2. For any tree $T$

$$
W(T)=\sum_{u \sim v \in E(G)} w(u, v) w(v, u)=\sum_{u<v, u, v \in V(T)} d(u, v) .
$$

For instance, this theorem was the basis for efficient computation of the Wiener index for trees [10]. Wiener never applied his index to connected graphs that are not trees. So one can extend his definition to graphs arbitrarily, the only restriction is that it should behave as the Wiener index on trees. 
The invariant:

$$
S z(G)=\sum_{u \sim v \in E(G)} w(u, v) w(v, u)
$$

is called the Szeged index of a graph [6]. In [15] M. Randic proposed a modification of the Szeged index and called the resulting index the revised Wiener index. However, we feel the newly described index arises from the Szeged index and therefore should be called the revised Szeged index $S z^{*}(G)$. Let $o(u, v)=o(v, u)$ denote the number of vertices of the same distance from $u$ and from $v$ :

$$
o(u, v)=|\{x \in V(G) \mid d(u, x)=d(v, x)\}|=|O(u, v)|
$$

The revised Szeged index is defined as follows:

$$
S z^{*}(G)=\sum_{u \sim v \in E(G)}(w(u, v)+(1 / 2) o(u, v))(w(v, u)+(1 / 2) o(u, v)) .
$$

In this note we study relationships among the Wiener index, the Szeged index and the revised Szeged index. Independent proofs of some of the results that were obtained by Dobrynin and Gutman [4] are presented.

\section{Edge Contributions}

If we compare the three indices: $W(G), S z(G)$ and $S z^{*}(G)$ we see that the Szeged and the revised Szeged index can be naturally described as a sum over the corresponding edge contributions. For an edge $e=u \sim v$ define:

$$
s(e)=w(u, v) w(v, u)
$$

and

$$
s^{*}(e)=(w(u, v)+(1 / 2) o(u, v))(w(v, u)+(1 / 2) o(u, v)) .
$$

Then

$$
S z(G)=\sum_{e \in E(G)} s(e)
$$

and

$$
S z^{*}(G)=\sum_{e \in E(G)} s^{*}(e) .
$$

In what follows here we try to mimic the edge-contribution for the Wiener index. Let $a$ and $b$ be two vertices of graph $G$ and let $p(a, b)$ denote the number of shortest paths in $G$ between $a$ and $b$ and let $k(a, b, e)$ be the number of shortest paths between $a$ and $b$ passing through the edge $e$. Define the edge contribution $w(e)$ as

$$
w(e):=\sum_{a<b, a, b \in V(G)} k(a, b, e) / p(a, b)
$$

\section{Lemma 2.1.}

$$
d(a, b)=\sum_{e \in E(G)} k(a, b, e) / p(a, b)
$$




\section{Theorem 2.2.}

$$
\sum_{e \in E(G)} w(e)=W(G)
$$

Proof.

$$
\begin{aligned}
\sum_{e \in E(G)} w(e) & =\sum_{e \in E(G)} \sum_{a<b, a, b \in V(G)} k(a, b, e) / p(a, b)= \\
& =\sum_{a<b, a, b \in V(G)} \sum_{e \in E(G)} k(a, b, e) / p(a, b)= \\
& =\sum_{a<b, a, b \in V(G)} d(a, b)=W(G) .
\end{aligned}
$$

\section{Results}

Let us present the results from [12].

Theorem 3.1. For a connected graph $G$ we have

$$
S z(G) \leq S z^{*}(G)
$$

The equality holds if and only if $G$ is bipartite.

The proof obviously follows from the fact the $s(e) \leq s^{*}(e)$ for each edge $e$.

Theorem 3.2. For a tree $T$ the three indices are the same:

$$
W(T)=S z(T)=S z^{*}(T)
$$

For general graphs the difference between the revised Szeged index and the original Szeged index may be quite large. Take for instance the complete graph $K_{n}$. The revised Szeged index is in this case equal to $S z^{*}\left(K_{n}\right)=n^{3}(n-1) / 8$ while $W(G)=S z(G)=n(n-1) / 2$. The quotient between the revised Szeged index and the original index is hence $n^{2}$.

This example shows that the leftmost equality may hold even for graphs that are not trees. However, it would be interesting to investigate the graphs, for which $W(G)=$ $S z^{*}(G)$. This equality clearly holds for trees. It would be interesting to know if such an equality may hold for any other graphs.

In a similar way one can compute the Szeged and the revised Szeged index for a cycle graph $C_{n}: S z\left(C_{n}\right)=n(\lfloor(n / 2)\rfloor)^{2}$ and $S z^{*}\left(C_{n}\right)=n^{3} / 4$. The Wiener index for cycles is $W\left(C_{n}\right)=n^{3} / 8$ for even $n$ and $W\left(C_{n}\right)=\left(n^{2}-1\right) n / 8$ for odd values of $n$; see [19].

In [5] the authors have used symmetry of graphs in order to simplify the calculation of the Wiener index of a graph. In [19], the process has been repeated for the Szeged index, see also [12] for corrections of some errors.

Now we explore the relationship between $w(e)$ and $s(e)$. 
Lemma 3.3. For every connected graph $G$ and for every edge $e=u \sim v$ it follows that

$$
w(e) \leq s(e)
$$

The equality holds if and only if $e$ is the only edge between $W(u, v)$ and $W(v, u)$ and each vertex $w \in O(u, v)$ adjacent to some vertex from $W(u, v) \cup W(v, u)$ determines a triangle $K_{3}$ on $\{u, v, w\}$.

Proof.

$$
\begin{aligned}
w(e) & :=\sum_{a \in V(G), b \in V(G)} k(a, b, e) / p(a, b) \\
& \leq \sum_{a \in W(u, v), b \in W(v, u)} k(a, b, e) / p(a, b) \\
& \leq \sum_{a \in W(u, v), b \in W(v, u)} 1=w(u, v) w(v, u)=s(e) .
\end{aligned}
$$

The tricky part is to determine when

$$
w(e)=s(e)
$$

The leftmost inequality becomes equality if and only if the existence of a shortest path between $a$ and $b$ passing through $e$ implies that all shortest paths from $a$ to $b$ pass through $e$. The second inequality turning into equality implies that for each vertex $a$ from $W(u, v)$ and for each vertex $b$ from $W(v, u)$ at least one shortest path between them passes through $e$. This implies that $e$ is the only edge joining $W(u, v)$ with $W(v, u)$. If $O(u, v)$ is nonempty, it must have a vertex $w$ that is connected both to $W(u, v)$ and $W(v, u)$ and forms an odd cycle including the edge $e$. Let $u^{\prime} \in W(u, v)$ be adjacent to $w$ and let $v^{\prime} \in W(v, u)$ be adjacent to $w$. The distance between $u^{\prime}$ and $v^{\prime}$ is 2 and the shortest path does not involve $e$. This means the equality cannot hold if $O(u, v)$ is nonempty.

Using the above Lemma one can prove the following inequality that was proven already in [9], see also [4].

Theorem 3.4. For any connected graph $G$ we have

$$
W(G) \leq S z(G)
$$

The equality holds for graphs with the following property. $G$ is obtained from complete graphs by vertex identifications. Two maximal complete graphs have at most one vertex in common. Each cycle lies in a complete graph.

Corollary 3.5. For any connected graph $G$ we have

$$
W(G)=S z^{*}(G)
$$

if and only if $G$ is a tree. 


\section{Arboreality of a graph}

In [12] the quotient $\beta(G)=S z(G) / S z^{*}(G)$ is considered as a measure of bipartivity of a graph. Since $0 \leq S z(G) / S z^{*}(G) \leq 1$ and $S z(G) / S z^{*}(G)=1$ only for bipartite graphs, it measures how close to a bipartite graph a given graph is. The question can be asked what is the minimum value of $\beta(G)$ for some classes of graphs.

There are two quotients that we may consider in a similar way:

$$
\alpha(G)=W(G) / S z(G)
$$

and

$$
\tau(G)=W(G) / S z^{*}(G)
$$

While $\alpha(G)$ measures how far from a tree composed of complete graphs is $G, \tau(G)$ measures the departure of $G$ from a tree. Let us call it arboreality of $G$. We may say that low $\alpha(G)$ means that $G$ is hollow, while large values mean it is dense. Obviously, $\alpha(G) \beta(G)=\tau(G)$ for any graph $G$; see [14] for an alternative attempt to address the issue of "tree-likeness".

The following tables list some values of these parameters for certain graphs. Numeric values were obtained by using VEGA system [11]. First we look at the complete graphs $K_{n}$.

\begin{tabular}{||r|r|r|l|r|l|l||}
\hline$G$ & $W(G)$ & $S z(G)$ & $S z^{*}(G)$ & $\alpha(G)$ & $\beta(G)$ & \multicolumn{1}{|c||}{$\tau(G)$} \\
\hline$K_{n}$ & $n(n-1) / 2$ & $n(n-1) / 2$ & $n^{3}(n-1) / 8$ & 1 & \multicolumn{1}{|c||}{$4 / n^{2}$} & \multicolumn{1}{|c|}{$4 / n^{2}$} \\
$K_{2}$ & 1 & 1 & 1.00 & 1 & 1.0 & 1.0 \\
$K_{3}$ & 3 & 6.75 & 1 & 0.444444 & 0.444444 \\
$K_{4}$ & 6 & 6 & 24.00 & 1 & 0.25 & 0.25 \\
$K_{5}$ & 10 & 62.50 & 1 & 0.16 & 0.16 \\
$K_{6}$ & 15 & 15 & 135.00 & 1 & 0.111111 & 0.111111 \\
$K_{7}$ & 21 & 21 & 257.25 & 1 & 0.0816327 & 0.0816327 \\
$K_{8}$ & 28 & 28 & 448.00 & 1 & 0.0625 & 0.0625 \\
$K_{9}$ & 36 & 36 & 729.00 & 1 & 0.0493827 & 0.0493827 \\
\hline
\end{tabular}

Next we consider complete bipartite graphs $K_{n, n}$

\begin{tabular}{||c|r|r|r|l|r|l||}
\hline$G$ & $W(G)$ & $S z(G)$ & $S z^{*}(G)$ & $\alpha(G)$ & $\beta(G)$ & \multicolumn{1}{|c||}{$\tau(G)$} \\
\hline$K_{n, n}$ & $n(3 n-2)$ & $n^{4}$ & $n^{4}$ & $(3 n-2) / n^{3}$ & 1 & $(3 n-2) / n^{3}$ \\
$K_{1,1}$ & 1 & 1 & 1 & 1.0 & 1 & 1.0 \\
$K_{2,2}$ & 8 & 16 & 16 & 0.5 & 1 & 0.5 \\
$K_{3,3}$ & 21 & 81 & 81 & 0.259259 & 1 & 0.259259 \\
$K_{4,4}$ & 40 & 256 & 256 & 0.15625 & 1 & 0.15625 \\
$K_{5,5}$ & 65 & 625 & 625 & 0.104 & 1 & 0.104 \\
$K_{6,6}$ & 96 & 1296 & 1296 & 0.0740741 & 1 & 0.0740741 \\
$K_{7,7}$ & 133 & 2401 & 2401 & 0.0553936 & 1 & 0.0553936 \\
$K_{8,8}$ & 176 & 4096 & 4096 & 0.0429688 & 1 & 0.0429688 \\
$K_{9,9}$ & 225 & 6561 & 6561 & 0.0342936 & 1 & 0.0342936 \\
$K_{10,10}$ & 280 & 10000 & 10000 & 0.028 & 1 & 0.028 \\
\hline
\end{tabular}


In the next table are the hypercube graphs.

\begin{tabular}{||r|r|r|r|l|r|l||}
\hline$G$ & $W(G)$ & $S z(G)$ & $S z^{*}(G)$ & $\alpha(G)$ & $\beta(G)$ & \multicolumn{1}{|c||}{$\tau(G)$} \\
\hline$Q_{n}$ & $n 2^{2(n-1)}$ & $n 2^{3(n-1)}$ & $n 2^{3(n-1)}$ & $2^{-(n-1)}$ & 1 & $2^{-(n-1)}$ \\
$Q_{1}$ & 1 & 1 & 1 & 1.0 & 1 & 1.0 \\
$Q_{2}$ & 8 & 16 & 16 & 0.5 & 1 & 0.5 \\
$Q_{3}$ & 48 & 192 & 192 & 0.25 & 1 & 0.25 \\
$Q_{4}$ & 256 & 2048 & 2048 & 0.125 & 1 & 0.125 \\
$Q_{5}$ & 1280 & 20480 & 20480 & 0.0625 & 1 & 0.0625 \\
$Q_{6}$ & 6144 & 196608 & 196608 & 0.03125 & 1 & 0.03125 \\
\hline
\end{tabular}

In the next table with paths, only 1's appear in the columns $\alpha(G), \beta(G)$, and $\tau(G)$ because all paths are trees.

\begin{tabular}{|c|r|r|r|r|r|r||}
\hline$G$ & $W(G)$ & $S z(G)$ & $S z^{*}(G)$ & $\alpha(G)$ & $\beta(G)$ & $\tau(G)$ \\
\hline$P_{n}$ & $\left(n^{3}-n\right) / 6$ & $\left(n^{3}-n\right) / 6$ & $\left(n^{3}-n\right) / 6$ & 1 & 1 & 1 \\
$P_{2}$ & 1 & 1 & 1 & 1 & 1 & 1 \\
$P_{3}$ & 4 & 4 & 4 & 1 & 1 & 1 \\
$P_{4}$ & 10 & 10 & 10 & 1 & 1 & 1 \\
$P_{5}$ & 20 & 20 & 20 & 1 & 1 & 1 \\
$P_{6}$ & 35 & 35 & 35 & 1 & 1 & 1 \\
$P_{7}$ & 56 & 56 & 56 & 1 & 1 & 1 \\
$P_{8}$ & 84 & 84 & 84 & 1 & 1 & 1 \\
$P_{9}$ & 120 & 120 & 120 & 1 & 1 & 1 \\
$P_{10}$ & 165 & 165 & 165 & 1 & 1 & 1 \\
\hline
\end{tabular}

Cycles.

\begin{tabular}{|c|c|c|c|c|c|c|}
\hline$G$ & $W(G)$ & $S z(G)$ & $S z^{*}(G)$ & $\alpha(G)$ & $\beta(G)$ & $\tau(G)$ \\
\hline$C_{n}, n$ even & $\frac{n^{3}}{8}$ & $\frac{n^{3}}{4}$ & $\frac{n^{3}}{4}$ & 0.5 & 1.0 & 0.5 \\
\hline$C_{n}, n$ odd & $\underline{n^{3}-n}$ & $\underline{n(n-1)^{2}}$ & $n^{3}$ & $n+1$ & $\underline{(n-1)^{2}}$ & $\underline{n^{2}-1}$ \\
\hline$C_{3}$ & ${ }^{8} 3$ & $\begin{array}{ll}4 & 4\end{array}$ & $6.75^{4}$ & $1.0^{2 n-2}$ & $\begin{array}{c}n^{2} \\
0.444444\end{array}$ & $\begin{array}{c}2 n^{2} \\
0.444444\end{array}$ \\
\hline$C_{4}$ & 8 & 16 & 16.0 & 0.5 & 1.0 & 0.5 \\
\hline$C_{5}$ & 15 & 20 & 31.25 & 0.75 & 0.64 & 0.48 \\
\hline$C_{6}$ & 27 & 54 & 54.0 & 0.5 & 1.0 & 0.5 \\
\hline$C_{7}$ & 42 & 63 & 85.75 & 0.666667 & 0.734694 & 0.489796 \\
\hline$C_{8}$ & 64 & 128 & 128.0 & 0.5 & 1.0 & 0.5 \\
\hline$C_{9}$ & 90 & 144 & 182.25 & 0.625 & 0.790123 & 0.493827 \\
\hline$C_{10}$ & 125 & 250 & 250.0 & 0.5 & 1.0 & 0.5 \\
\hline$C_{11}$ & 165 & 275 & 332.75 & 0.6 & 0.826446 & 0.495868 \\
\hline$C_{12}$ & 216 & 432 & 432.0 & 0.5 & 1.0 & 0.5 \\
\hline$C_{13}$ & 273 & 468 & 549.25 & 0.583333 & 0.852071 & 0.497041 \\
\hline$C_{14}$ & 343 & 686 & 686.0 & 0.5 & 1.0 & 0.5 \\
\hline$C_{15}$ & 420 & 735 & 843.75 & 0.571429 & 0.871111 & 0.497778 \\
\hline
\end{tabular}


Some generalized Petersen graphs.

\begin{tabular}{||c|r|r|r|r|l|l||}
\hline graph & $W(G)$ & $S z(G)$ & $S z^{*}(G)$ & $\alpha(G)$ & $\beta(G)$ & \multicolumn{1}{|c||}{$\tau(G)$} \\
\hline$P(3,1)$ & 21 & 51 & 81.0 & 0.411765 & 0.62963 & 0.259259 \\
$P(4,1)$ & 48 & 192 & 192.0 & 0.25 & 1.0 & 0.25 \\
$P(5,1)$ & 85 & 285 & 375.0 & 0.298246 & 0.76 & 0.226667 \\
$P(5,2)$ & 75 & 135 & 375.0 & 0.555556 & 0.36 & 0.2 \\
$P(6,1)$ & 144 & 648 & 648.0 & 0.222222 & 1.0 & 0.222222 \\
$P(6,2)$ & 135 & 354 & 634.5 & 0.381356 & 0.55792 & 0.212766 \\
$P(7,1)$ & 217 & 847 & 1029.0 & 0.256198 & 0.823129 & 0.210884 \\
$P(7,2)$ & 189 & 602 & 1029.0 & 0.313953 & 0.585034 & 0.183673 \\
$P(8,1)$ & 320 & 1536 & 1536.0 & 0.208333 & 1.0 & 0.208333 \\
$P(8,2)$ & 280 & 856 & 1528.0 & 0.327103 & 0.560209 & 0.183246 \\
$P(8,3)$ & 272 & 1536 & 1536.0 & 0.177083 & 1.0 & 0.177083 \\
\hline
\end{tabular}

The following comparison of the three indices is taken from Pisanski and Randic [12].

\begin{tabular}{||c|c|c|c||}
\hline & Wiener index & Szeged Index & Revised Szeged index \\
\hline source & {$[17]$} & {$[6]$} & {$[15]$} \\
\hline notation & $W(G)$ & $S z(G)$ & $S z^{*}(G)$ \\
\hline$G=P_{n}$ & $\left(n^{3}-n\right) / 6$ & $\left(n^{3}-n\right) / 6$ & $\left(n^{3}-n\right) / 6$ \\
\hline$G=C_{n}, n$ even & $n^{3} / 8$ & $n^{3} / 4$ & $n^{3} / 4$ \\
\hline$G=C_{n}, n$ odd & $\left(n^{3}-n\right) / 8$ & $n(n-1)^{2} / 4$ & $n^{3} / 4$ \\
\hline$G=K_{n}$ & $n(n-1) / 2$ & $n(n-1) / 2$ & $n^{3}(n-1) / 8$ \\
\hline$G=H \square K$ & $W(K)|V(H)|^{2}+$ & $W(K)|V(H)|^{3}+$ & $W(K)|V(H)|^{3}+$ \\
& $+W(H)|V(K)|^{2}$ & $+W(H)|V(K)|^{3}$ & $+W(H)|V(K)|^{3}$ \\
\hline$G{ }^{k}$ & $k W(G)|V(G)|^{2(k-1)}$ & $k S z(G)|V(G)|^{3(k-1)}$ & $k S z^{*}(G)|V(G)|^{3(k-1)}$ \\
\hline$G=Q_{n}$ & $n 2^{2(n-1)}$ & $n 2^{3(n-1)}$ & $n 2^{3(n-1)}$ \\
\hline$G=K_{n, n, \ldots, n}$ & $n r(n r+n-2) / 2$ & $r(r-1) n^{4} / 2$ & $r^{3}(r-1) n^{4} / 8$ \\
\hline$G$ bipartite & $W(G)$ & $S z(G)$ & $S z(G)$ \\
\hline$T$ tree & $W(T)$ & $W(T)$ & $W(T)$ \\
\hline
\end{tabular}

\section{Conclusion}

There is a natural difference between the Wiener index and the other indices described above. For the Wiener index one may usually compute the contribution of each vertex and then sum the contributions. In the Szeged index one may compute an edge contribution and then sum the obtained edge contributions. The same is true for the revised Szeged index. However, this distinction is not absolute. The latter approach may be used for the Wiener index as shown in [13] however, the computations are more involved. We hope we can use this approach to study more closely the relationship between the indices $W(G), S z(G)$ and $S z^{*}(G)$.

In [2] the authors have computed the Wiener index and the Szeged index for benzenoid graphs in linear time. It is clear that their methods give also the revised Szeged index since all benzenoids are bipartite; see also [1, 3, 7]. It is well-known that on trees, the Wiener index can be computed in linear time [10], and, consequently, the Szeged indices on trees can be computed in linear time. In general, the Wiener and Szeged indices can 
be computed in time $O(m n)$ [18]. The revised Szeged index can be computed within the same time complexity by a straightforward method using the distance matrix. The question is whether the computation of the revised Szeged index can be done faster for some classes of graphs.

\section{Acknowledgements}

The authors would like to thank Sandi Klavžar and Milan Randić for careful reading of the manuscript and many useful suggestions. The authors acknowledge partial funding of this research via ARSS of Slovenia, grants: L2-7230, P1-0294, L2-7207 and the PASCAL network of excellence in the 6th framework.

\section{References}

[1] J. Brunvoll, B. N. Cyvin and S. J. Cyvin, Enumeration and Classification of Coronoid Hydrocarbons, J. Chem. Inf. Comput. Sci. (1987) 27, 14-21.

[2] V. Chepoi and S. Klavžar, The Wiener index and the Szeged index of benzenoid systems in linear time, J. Chem. Inf. Comput. Sci. 37 (1997), 752-755.

[3] A. A. Dobrynin, Graphs Having the Maximal Value of the Szeged Index, Croatica Chemica Acta 70 (1997) 819-825.

[4] A. A. Dobrynin and I. Gutman, Solving a problem connected with distances in graphs, Graph Theory Notes New York XXVIII (5) (1994), 21-23.

[5] A. Graovac and T. Pisanski, On the Wiener index of a graph, J. Math. Chem. 8 (1991), 53-62.

[6] I. Gutman, A formula for the Wiener number of trees and its extension to graphs containing cycles, Graph Theory Notes New York 27 (1994), 9-15.

[7] I. Gutman and S. Klavžar, A method for calculating Wiener numbers of benzenoid hydrocarbons, Acta Chim. Hung. 133 (1996), 389-399.

[8] W. Imrich and S. Klavžar, Product graphs, Wiley-Interscience, New York, 2000.

[9] S. Klavžar, A. Rajapakse and I. Gutman, The Szeged and the Wiener index of graphs, Appl. Math. Lett. 9 (1996), 45-49.

[10] B. Mohar and T. Pisanski, How to compute the Wiener index of a graph, J. Math. Chem. 2 (1988), 267-277.

[11] T. Pisanski \& coworkers, VEGA, a programming tool for manipulating discrete mathematical structures, see http://vega.ijp.si.

[12] T. Pisanski and M. Randić, Use of the Szeged index and the revised Szeged index for measuring network bipartivity, Discrete Appl. Math., to appear.

[13] T. Pisanski and J. Žerovnik, Weights on edges of chemical graphs determined by paths, $J$. Chem. Inf. Comput. Sci. 34 (1994), 395-397.

[14] M. Randić, Characterization of molecular branching, J. Am. Chem. Soc. 97 (1975), 6609-6615.

[15] M. Randić, On generalization of Wiener index for cyclic structures, Acta Chimica Slovenica 49 (2002), 483-496.

[16] N. Trinajstić, Chemical graph theory, 2nd rev. ed., CRC Press, Boca Raton, 1992.

[17] H. Wiener, Correlation of heats of isomerization, and differences in heats of vaporization of isomers, among the paraffin hydrocarons, J. Am. Chem. Soc. 69 (1947), 2636-2638.

[18] J. Žerovnik, Computing the Szeged index, Croatica Chemica Acta 69 (1996), 837-843. 
[19] J. Žerovnik, Szeged index of symmetric graphs, J. Chem. Inf. Comput. Sci., 39 (1999), 77-80. 